\title{
A I2-year follow-up study of combined treatment of post-severe acute respiratory syndrome patients with femoral head necrosis
}

This article was published in the following Dove Press journal:

Therapeutics and Clinical Risk Management

19 October 2017

Number of times this article has been viewed

\author{
Tiansheng Liu'1,2 \\ Jinchao $\mathrm{Ma}^{3}$ \\ $\mathrm{Bin} \mathrm{Su}^{4}$ \\ Hao Wang ${ }^{3}$ \\ Qi Wang ${ }^{3}$ \\ Xinlong $\mathrm{Ma}^{1,2}$
}

'Department of Orthopaedic Surgery,

The General Hospital of Tianjin

Medical University, ${ }^{2}$ Department of

Orthopaedic Surgery, Tianjin Hospital,

${ }^{3}$ Department of Orthopaedic Surgery,

${ }^{4}$ Department of Pneumology, the

Affiliated Hospital of Logistics

University of PAP, Tianjin, People's

Republic of China
Correspondence: Xinlong Ma

Department of Orthopaedic Surgery,

The General Hospital of Tianjin

Medical University, No I54 Anshandao,

Heping District, Tianjin 300000,

People's Republic of China

Tel +8622 28332917

Email landslands2003@sina.com
Objective: To investigate the long-term efficacy of a combination treatment of alendronate, extracorporeal shock and hyperbaric oxygen for osteonecrosis of the femoral head (ONFH) of post-severe acute respiratory syndrome (SARS) patients.

Patients and methods: The retrospective study was performed including a total of 37 postSARS ONFH patients (66 hip joints) in the Department of Orthopedics of the General Hospital of Tianjin Medical University between November 2003 and November 2015, consisting of 6 males (11 hip joints) and 31 females ( 55 hip joints), with age between 19 and 47 years (average 29.9 years). Visual analog scale (VAS) score, Harris score and Association Research Circulation Osseous (ARCO) stage of imaging examination were compared among those before treatment, and at 1, 3, 6, 9 and 12 years after treatment. Paired $t$-test was used for statistical analysis of VAS and Harris score before and after treatment. Difference of effective rate on all stages was analyzed with Chi-square test.

Results: With 12-year follow-up, significant improvements on VAS (6.81 of pre-treatment vs 3.94 of 12-year post-treatment) and Harris score (74.54 of pre-treatment vs 80.14 of 12-year post-treatment) were observed (all $p<0.05$ ). Effective rate showed statistical significance among three stages of ARCO $(p<0.05)$. The combined treatment showed different efficacies on different ARCO stages; the best was on ARCO Phase I.

Conclusion: The combined treatment may delay or discontinue the development of ONFH in post-SARS patients.

Keywords: SARS, osteonecrosis of the femoral head, alendronate, extracorporeal shock wave, hyperbaric oxygen

\section{Introduction}

Osteonecrosis of the femoral head (ONFH) is a refractory disease in orthopedics practice and can be divided into traumatic and non-traumatic types. Major reasons causing non-traumatic ONFH include hormone abuse, ethanol overdosage, congenital and other unknown factors. In the spring of 2003, the severe acute respiratory syndrome (SARS) broke out in most cities of China, and the patients who recovered from SARS suffered mostly from the refractory disease - ONFH. The incidence of ONFH is $\sim 60 \%-70 \%$ after SARS treatment, and ONFH mostly occurred in bilateral sides. ${ }^{1}$ It is thought that post-SARS ONFH is mainly caused by the application of high dosage of glucocorticoid hormones during SARS treatment, though some studies indicated that other reasons might be applicable in those cases. ${ }^{2,3}$ SARS could be one independent risk factor causing $\mathrm{ONFH},{ }^{4,5}$ perhaps via mediation by $\mathrm{S}$ proteins. ${ }^{6,7}$ 
Due to unclear pathogenesis and mechanism of SARS, there has been no definitive treatment strategy for ONFH. Major treatment approaches include non-surgery (drugs, hyperbaric oxygen, extracorporeal shock wave, pulsed electromagnetic treatment, decreased loading, and proper breaking and retraction) and surgery (medullary decompression of femur, bone transplant without vessels, osteotomy, autograft bone transplantation with vessels, and artificial joint replacement). ${ }^{8-10}$ Here, we retrospectively studied the long-term efficacies of the combined treatment (non-surgery) for 37 post-SARS patients suffering from ONFH between the end of 2003 and the end of 2015.

\section{Patients and methods} General information

This is a retrospective study. A total of 44 post-SARS ONFH patients (including 74 femoral joints) were recruited between November 2013 and November 2015. Inclusive criteria: 1) matching diagnostic guideline of $\mathrm{ONFH} ;{ }^{10} 2$ ) with history of SARS infection; 3) received combined treatment including alendronate, extracorporeal shock wave, and hyperbaric oxygen; and 4) obtained patient's written consents. Exclusive criteria: 1) traumatic ONFH; 2) atypical imaging manifestation or not matching diagnostic guideline; and 3) surgery treatment. This study has been approved by the ethical committee of Tianjin Hospital.

\section{Judging criteria}

Degree of clinical pain was evaluated based on visual analog scale (VAS) approach. Harris score, as a standard evaluation to various hip disabilities and methods of treatment (full mark =100), was used for assigning good, satisfactory, acceptable and unfavorable conditions as higher than 89 , 80-89, 70-79 and $<70$, respectively. Imaging examination was performed on all patients. Volume and volume ratio of femoral necrosis were calculated based on magnetic resonance imaging (MRI) images. ${ }^{11}$ All patients were examined with imaging techniques. The degree of ONFH shown on imaging was graded according to Association Research Circulation Osseous (ARCO) staging criteria. The volume and volume ratio of femoral necrosis were analyzed based on MRI images by using a published calculating method. ${ }^{11}$ By comparing with actual measurement from surgical specimens, the MRI image-based calculation was demonstrated to be close to actual values.

\section{Treatment approach}

All patients included in this study received the following treatment: 1) $70 \mathrm{mg}$ weekly oral application of alendronate sodium for 12 consecutive years; 2) extracorporeal shock wave treatment was performed using HK.ESWL-Vm equipment (Huikang Med, Shenzhen, China). In brief, patients were fixed in a supine position with localization of the femoral necrosis region by scanning. Using $0.18-0.25 \mathrm{~mJ} / \mathrm{mm}^{2}$ energy density, 2-3 shocking sites were selected in single treatment for 1,000 times of shocking (60 per min). One treatment course thus consisted of 20,000-30,000 shocks. A total of 5-8 courses were performed within 2-3 months; 3 ) hyperbaric oxygen was performed at 1.5 -fold of normal atmosphere pressure for $2 \mathrm{~h}$. After 100 treatments (once daily), treatment was performed twice per week, with 5-7 days rest after every 30 treatments. In our department, each patient was re-examined monthly on VAS, Harris score and femoral joint MRI examination, which were used to calculate volume ratio of $\mathrm{ONFH}$.

\section{Statistical processing}

Measurement data were presented as mean $\pm \mathrm{SD}$. Paired $t$-test was used to compare VAS score and Harris score before and after treatment. Chi-square test was used to compare effective rate of femoral necrosis between Stage I and Stage III in the combined treatment scenario. A statistical significance was defined when $p<0.05$. SPSS 17.0 (SPSS Inc., Chicago, IL, USA) software was used to analyze data.

\section{Results}

\section{Clinical information of patients}

During follow-up period, 1 patient received artificial hip joint replacement, and 6 patients were lost to follow-up. Eventually, there were 37 post-SARS ONFH patients (66 hip joints) included, consisting of 6 males (11 hip joints) and 31 females (55 hip joints). All patients received prednisone treatment for $4-11$ days (average $=9.56 \pm 3.70$ days), with a daily dosage of $180-1,430 \mathrm{mg}$ (average $=545.00 \pm 366.17 \mathrm{mg}$ ) and total dosage between 1,189 and 11,440 mg (average $=4,702.22 \pm 2,826.94 \mathrm{mg}$ ). The average age of patients at disease onset was 29.9, ranging from 19 to 47 years. Among 37 patients, there were 29 bilateral necrosis and 8 unilateral cases. There were 14 hip joints with limb shortening by $1-2 \mathrm{~cm}$. All patients presented hip joint pain to different degrees. VAS score showed 4 patients ( 7 hip joints) with $<5$ points, 10 patients (17 joints) at 5-7 points and 23 patients (42 joints) at 8-10 points. Harris score showed 4 cases ( 7 joints) with good, 5 cases ( 8 joints) with satisfactory, 10 cases (19 joints) with acceptable and 18 cases (32 joints) with unfavorable conditions. Imaging examination of ARCO stage ${ }^{12}$ showed 36, 21 and 9 joints at Phase I, Phase II and Phase III, respectively. 


\section{Comparison of VAS and Harris score in post-SARS patients}

Within a 12-year follow-up period, patients showed, in general, significant improvements on VAS score, as averaged VAS score decreased from $6.81 \pm 1.70$ before treatment to $6.43 \pm 1.66$ at 1 year afterward (first year Student's $t$-test $[\mathrm{t} 1 \mathrm{a}]=2.578, p<0.01)$. Averaged VAS score was further lowered with elongated treatment time, reaching $6.35 \pm 1.51$ at the 3rd year (3rd year Student's $t$-test $[\mathrm{t} 2 \mathrm{a}]=2.750, p<0.05$ ) and $6.00 \pm 1.68$ at the 6 th year (6th year Student's $t$-test $[\mathrm{t} 3 \mathrm{a}]=3.274, p<0.05), 4.92 \pm 1.82$ at the 9 th year $(9$ th year Student's $t$-test [t4a] $=5.845, p<0.05$ ) and eventually $3.97 \pm 2.23$ at the 12 th year at the end of observation window (12th year Student's $t$-test [t5a] $=6.844, p<0.05$ ).

Harris score showed gradual improvement with continuous drug treatment. Before recruitment, averaged Harris score of all patients was $74.54 \pm 9.92$, which was elevated to $76.49 \pm 8.75$ (first year Student's $t$-test [t1b] $=2.499$, $p<0.05) 1$ year after, and further increased to $77.11 \pm 8.33$ at the 3 rd year (3rd year Student's $t$-test [t2b] $=2.779$, $p<0.05$ ), 77.92 \pm 8.01 at the 6th year (6th year Student's $t$-test $[\mathrm{t} 3 \mathrm{~b}]=2.857, p<0.05), 78.78 \pm 8.79$ at the 9 th year (9th year Student's $t$-test [t4b] $=3.138, p<0.05$ ) and $80.14 \pm 9.47$ at the 12 th year (12th year Student's $t$-test $[\mathrm{t} 5 \mathrm{~b}]=3.590, p<0.05$ ). In summary, patient VAS and Harris score were all significantly improved after 12-year treatment (Table 1).

\section{Femoral head necrosis ratio by MRI examination}

All 37 patients (with 66 hips) were staged based on the ARCO guideline. Overall effective rate of 12-year treatment was calculated and compared among different stages. Those with enlarged necrosis volume ratio were identified as ineffective treatment, while those with unchanged or shrink volume ratio were designated as effective treatment. Among 36 hips of Stage I, effective rate was $91.67 \%$; such figures, however, were $80.95 \%$ among 21 hips at Stage II, and only $55.56 \%$ within 9 hips at Stage III. The overall effective rate was $83.33 \%$. Statistical analysis showed that combined therapy has different effectiveness treating femoral head necrosis at
Stage I to Stage III, with the highest effective rate in Stage I patients $\left(\chi^{2}=6.781, p<0.05\right)$. The improvements included the alleviation of pain and lameness and the partial recovery of hip joint functions, such as buckling and abduction. The analysis about volume ratio of femoral head bones based on MRI image indicated that enlarged volume ratio presented ineffective treatment. It can effectively delay ONFH progression after SARS treatment (Figure 1A-F). Among all follow-up patients, 1 of them received left artificial hip joint replacement after 5 years (Figure 2); 2 patients presented digestive tract symptoms.

\section{Discussion}

In our study, the average age of post-SARS ONFH patients was 29.9 years. Due to more daily activity in young people leading to heavy load on hip joints, hip joint surgery in early stages may encounter the risk of resurgery. Stringent challenge may result in patient's psychological, physiological and economic burden once undergoing surgical failure or complications. Some surgeons thus suggested conserving the femoral heads maximally instead of artificial hip joint replacement. Before making a diagnosis based on imaging evidence showing femoral head collapse, conservation treatment mainly aims to recover the function of the hip joint, to alleviate pain, and to block or retard collapse progression for curing necrosis lesion, thus improving prognosis and reducing surgical risks.

Currently, major conservation treatments include drugs, hyperbaric oxygen, extracorporeal shock, pulsed electromagnetic treatment, decreased loading, and proper breaking and retraction. ${ }^{8-10}$ This study performed combined treatment including alendronate sodium, extracorporeal shock and hyperbaric oxygen on all included patients, followed by 12-year post-op follow-ups. Alendronate drugs can inhibit bone absorption ability of osteoclasts, accelerate osteoclast apoptosis, improve bone volume and bone strength, and facilitate bone healing, thus impeding and retarding ONFH progression, decreasing bone absorption and femoral head collapse, as well as decreasing the frequency of surgery for collapse. Hong et al showed that alendronate is one effective

Table I VAS and Harris score of 37 post-SARS ONFH patients before and after treatment

\begin{tabular}{lllllll}
\hline Score & Pre-treatment & \multicolumn{3}{l}{ Post-treatment } & & \\
\cline { 3 - 7 } & & $\mathbf{1}$ year & $\mathbf{3}$ years & $\mathbf{6}$ years & $\mathbf{9}$ years & $\mathbf{1 2}$ years \\
\hline VAS & $6.81 \pm 1.70$ & $6.43 \pm 1.66$ & $6.35 \pm 1.5 \mathrm{I}$ & $6.00 \pm 1.68$ & $4.92 \pm 1.82$ & $3.97 \pm 2.23$ \\
Harris & $74.54 \pm 9.92$ & $76.49 \pm 8.75$ & $77.11 \pm 8.33$ & $77.92 \pm 8.01$ & $78.78 \pm 8.79$ & $80.14 \pm 9.47$ \\
\hline
\end{tabular}

Notes: Data were presented as mean \pm standard error of means. For VAS score, the post-treatment values at I, 3, 6, 9 and I2 years were compared with pre-treatment, the $p$-values were $0.014,0.009,0.002,0.000$ and 0.000 , respectively; for Harris score, the post-treatment values at I, 3, 6, 9 and I2 years were compared with pre-treatment, the $p$-values were $0.017,0.009,0.007,0.003$ and 0.001 , respectively.

Abbreviations: ONFH, osteonecrosis of the femoral head; SARS, severe acute respiratory syndrome; VAS, visual analog scale. 

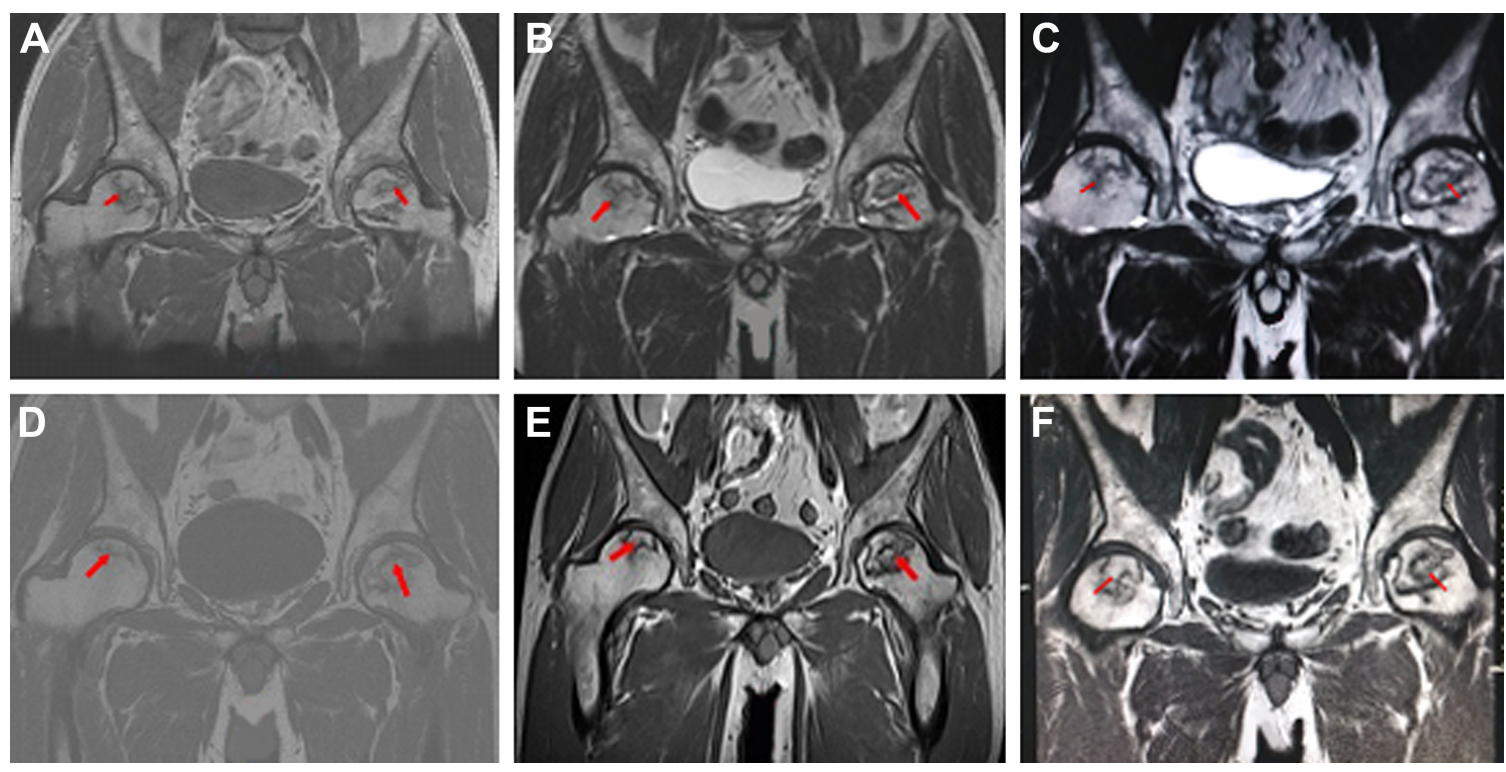

Figure I MRI images show the differences of femoral hip cystic lesions pre- and post-treatment from a 47-year-old male patient with bilateral femoral hip cystic lesions. Notes: A: pre-treatment; B: I year after treatment; C: 3 years after treatment; D: 6 years after treatment; E: 9 years after treatment and F: I 2 years after treatment. Red arrows point out the cystic lesions.

Abbreviation: MRI, magnetic resonance imaging.

method for treating ONFH and can reduce bone marrow edema. ${ }^{13}$ However, due to potential side effects including jaw bone necrosis or bone fracture after long-term application of alendronate, some surgeons still prefer surgery approaches. ${ }^{13}$ Bone et al observed 10-year study on menopausal women and found no significant correlation between alendronate drugs and bone fracture. ${ }^{14}$ This study performed 12-year alendronate sodium treatment (70 $\mathrm{mg}$ weekly) on all post-

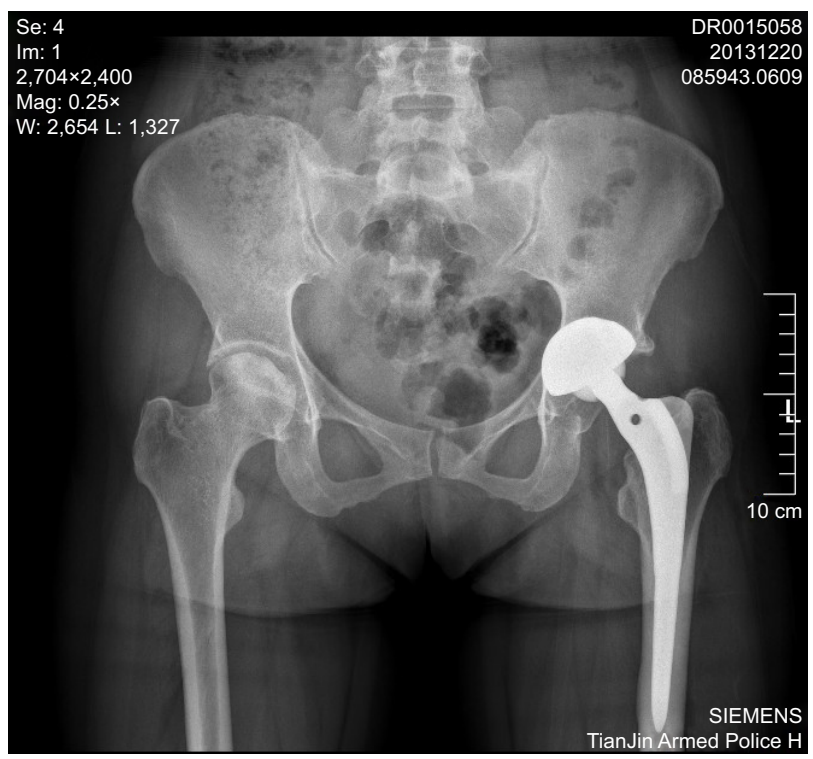

Figure 2 Pelvis images of bilateral hips from a 35 -year-old female patient who received left artificial hip joint replacement.

Note: Image shows pelvis $X$-ray film at 5 years after surgery, and the change of the left hip joint with flattening and sclerosis of the right femoral head.
SARS ONFH patients and found no jaw bone absorption or fracture among all patients. Extracorporeal shock wave therapy (ESWT) can alleviate or treat ONFH via enhancing expression of vascular endothelial growth factor (VEGF) in subchondral bones of the femoral head and induction of de novo angiogenesis to improve femoral head blood supply. Vulpiani et al found that ESWT could benefit bone reconstruction and regeneration via stimulating vascular growth factor expression. ${ }^{15,16}$ Wang et al compared ESWT and surgery approach and found that ESWT had significant clinical efficacy and less probability of surgery. ${ }^{17}$ Lin et al also showed efficacy of ESWT on prednisone treatmentinduced ONFH after systemic lupus erythematosus (SLE) treatment. ${ }^{18}$ Ludwig et al reported that 1-year ESWT treatment significantly alleviated pain, improved hip joint function and reduced bone necrosis lesions on ONFH patients at ARCO Phase I to Phase III. ${ }^{19}$ ESWT helps to treat ONFH mainly via inducing tissue regeneration of mesenchymal and extracellular reactions and inducing femoral head angiogenesis. We performed long-term follow-up on all patients receiving ESWT and showed that ESWT could retard disease worsening and improve clinical symptoms on early phase ONFH patients. Moreover, Camporesi et al performed a 7-year follow-up and showed that 6-week continuous hyperbaric oxygen treatment could significantly improve clinical symptoms of ONFH. ${ }^{20}$ Hyperbaric oxygen environment can enhance oxygen concentration in blood, thus alleviating oxygen perfusion into bone tissues for reducing 
bone marrow edema, thus reducing bone pressure, venous drainage and microcirculation. Moreover, hyperbaric oxygen can also stimulate vessel angiogenesis and the function of osteoblast and osteoclast cells, thus providing necessary pre-conditions for treating ONFH. Koren et al performed an 11.1 \pm 5.1 -year follow-up study and indicated that hyperbaric oxygen was 1 effective method for treating Phase I and Phase II ONFH. ${ }^{21}$ It is still debatable about the effects of single usage of hyperbaric oxygen to treat post-SARS ONFH. Wu et $\mathrm{al}^{22}$ performed a follow-up on Phase I ONFH of SARS patients and reported that hyperbaric oxygen cannot effectively impede or reverse progression of Phase I ONFH after SARS, while Qiu and Zhang ${ }^{3}$ attributed hyperbaric oxygen to be a satisfactory method for treating post-SARS ONFH. This study performed regular combined treatment consisting of alendronate drug, extracorporeal shock wave and hyperbaric oxygen. By 12-year long-term follow-up, some patients showed clinical improvement of ONFH, while most patients showed retardation of disease progression or even reversion. By statistical analysis of VAS and Harris score before and after treatment, we found significant differences $(p<0.05)$. Therefore, we believe that long-term treatment of combined therapy including alendronate drug, extracorporeal shock wave and hyperbaric oxygen could effectively retard ONFH progression, alleviate patient's clinical symptoms and improve hip joint function, thus decreasing the risk of surgery on post-SARS ONFH patients.

The current study has certain limitations. First, evaluation of treatment efficiency mainly depends on a routine body check, MRI examination and patient's interview, lacking precise pathology evidence. Second, we only compared treatment efficiency among 3 different approaches, but did not further analyze any difference between single and combined therapy.

\section{Conclusion}

In summary, post-SARS ONFH severely affects patients' health. Long-term combined treatment using alendronate drug, extracorporeal shock wave and hyperbaric oxygen can improve femoral head hemodynamics and osteogenesis, thus effectively managing progression of post-SARS ONFH, with significant clinical treatment efficacy, thus retarding or decreasing the risk of surgery. However, its detailed mechanism and the precise reason for post-SARS ONFH are still unclear and require further investigations.

\section{Disclosure}

The authors report no conflicts of interest in this work.

\section{References}

1. Liu Z, Li Z, Zhang N, Zhang Q, Cheng L, Sun W. [A prospective analysis of the progression of post-SARS bilateral femoral head necrosis]. Chin J Bone Joint Inj. 2010;25(10):889-893. Chinese.

2. Qiu M, Zhu M. [Post-SARS femoral head necrosis may be caused by factors other than glucocorticoid hormones]. Zhonghua Yi Xue Za Zhi. 2004;84(10):812. Chinese.

3. Qiu N, Zhang W. [Pathology and treatment of post-SARS femoral head necrosis]. Chin J Tissue Eng Res. 2013;17(30):5525-5530. Chinese.

4. Lv Y, Wan Y, Wu Y. [Prediction of SARS virus S protein at B cell epitope]. J Third Mil Med Univ. 2004;26(2):101-103. Chinese.

5. Hofmann H, Geier M, Marzi A, et al. Susceptibility to SARS coronavirus $\mathrm{S}$ protein-driven infection correlates with expression of angiotensin converting enzyme 2 and infection can be blocked by soluble receptor. Biochem Biophys Res Commun. 2004;319(4):1216-1221.

6. Ksiazek TG, Erdman D, Goldsmith CS, et al. A novel coronavirus associated with severe acute respiratory syndrome. $N$ Engl $J$ Med. 2003;348(20):1953-1966.

7. Dong X, Yang X, Li Y, et al. [Molecular cloning, expression, purification and immune property of SARS virus S2 gene]. Xi Bao Yu Fen Zi Mian Yi Xue Za Zhi. 2004;20(3):257-260. Chinese.

8. Wu B, Dong Z, Li S, Song H. Steroid-induced ischemic bone necrosis of femoral head: treatment strategies. Pak J Med Sci. 2015;31(2): $471-476$.

9. Tripathy SK, Goyal T, Sen RK. Management of femoral head osteonecrosis: current concepts. Indian J Orthop. 2015;49(1):28-45.

10. Li Z. [Clinical diagnostic guideline for femoral head necrosis (2015 edition)]. Chin J Joint Surg (Electronic Edition). 2015;(1):97-100. Chinese.

11. Hernigou P, Lambotte JC. Volumetric analysis of osteonecrosis of the femur. Anatomical correlation using MRI. J Bone Joint Surg Br. 2001;83(5):672-675.

12. Sugano N, Atsumi T, Ohzono K, Kubo T, Hotokebuchi T, Takaoka K. The 2001 revised criteria for diagnosis, classification, and staging of idiopathic osteonecrosis of the femoral head. J Orthop Sci. 2002; 7(5):601-605.

13. Hong YC, Luo RB, Lin T, Zhong HM, Shi JB. Efficacy of alendronate for preventing collapse of femoral head in adult patients with nontraumatic osteonecrosis. Biomed Res Int. 2014;2014:716538.

14. Bone HG, Hosking D, Devogelaer JP, et al. Ten years' experience with alendronate for osteoporosis in postmenopausal women. $N$ Engl J Med. 2004;350(12):1189-1199.

15. Wang CJ. Extracorporeal shockwave therapy in musculoskeletal disorders. J Orthop Surg Res. 2012;7:11.

16. Vulpiani MC, Vetrano M, Trischitta D, et al. Extracorporeal shock wave therapy in early osteonecrosis of the femoral head: prospective clinical study with long-term follow-up. Arch Orthop Trauma Surg. 2012;132(4):499-508.

17. Wang CJ, Huang CC, Wang JW, Wong T, Yang YJ. Long-term results of extracorporeal shockwave therapy and core decompression in osteonecrosis of the femoral head with eight- to nine-year follow-up. Biomed J. 2012;35(6):481-485.

18. Lin PC, Wang CJ, Yang KD, Wang FS, Ko JY, Huang CC. Extracorporeal shockwave treatment of osteonecrosis of the femoral head in systemic lupus erythematosus. J Arthroplasty. 2006;21(6):911-915.

19. Ludwig J, Lauber S, Lauber HJ, Dreisilker U, Raedel R, Hotzinger H. High-energy shock wave treatment of femoral head necrosis in adults Clin Orthop Relat Res. 2001;(387):119-126.

20. Camporesi EM, Vezzani G, Bosco G, Mangar D, Bernasek TL. Hyperbaric oxygen therapy in femoral head necrosis. $J$ Arthroplasty. 2010;25(6 suppl):118-123.

21. Koren L, Ginesin E, Melamed Y, Norman D, Levin D, Peled E. Hyperbaric oxygen for stage I and II femoral head osteonecrosis. Orthopedics. 2015;38(3):e200-e205.

22. Wu J, Jiang Q, Hong N, Chi Q. [A follow-up study of phase I femoral head ischemia necrosis on SARS patients after hyperbaric oxygen treatment]. Chin J Med Imag Technol. 2008;24(4):608-610. Chinese. 


\section{Publish your work in this journal}

Therapeutics and Clinical Risk Management is an international, peerreviewed journal of clinical therapeutics and risk management, focusing on concise rapid reporting of clinical studies in all therapeutic areas, outcomes, safety, and programs for the effective, safe, and sustained use of medicines. This journal is indexed on PubMed Central, CAS,

EMBase, Scopus and the Elsevier Bibliographic databases. The manuscript management system is completely online and includes a very quick and fair peer-review system, which is all easy to use. Visit http://www.dovepress.com/testimonials.php to read real quotes from published authors.

Submit your manuscript here: http://www.dovepress.com/therapeutics-and-clinical-risk-management-journal 